
\title{
25 Research Soure \\ The Biomechanical Role of the Fibula in Lower Limbs: A Fracture Mechanics Analysis
}

\section{Bin Shao}

Binzhou Medical University Hospital

Jianqiang Xing

Binzhou Medical College Affiliated Hospital: Binzhou Medical University Hospital

\section{Dong Liu}

Binzhou Medical University Hospital

\section{Dawei Wang}

Binzhou Medical University Hospital

\section{Bei Zhao}

Liaocheng People's Hospital

\section{Tingguang Wang}

The people hospital of zouping city

Weidong Mu ( $D$ Dr_muweidong@163.com )

Shandong Provincial Hospital

\section{Research article}

Keywords: Fracture mechanics, Fibular defect model, Biomechanics, LS-DYNA software

Posted Date: May 25th, 2021

DOl: https://doi.org/10.21203/rs.3.rs-508618/v1

License: (c) (i) This work is licensed under a Creative Commons Attribution 4.0 International License.

Read Full License 


\section{Abstract}

\section{Background}

Fibular grafting is widely used in the treatment of various bone nonunions and defects because of its good therapeutic effects. Furthermore, partial fibular resection has been used as a treatment for fibular tumors, injuries, and other conditions. The fibula plays important roles in the biomechanics of the lower limbs. Some experts have used cadaver specimens to study these aspects. In this study, the biomechanical effects of the fibula in lower limbs were researched through mechanics analysis.

\section{Methods}

We randomly collected knee joint computed tomography (CT) scans from eight adults, including three left knees and five right knees. The DICOM formatted CT scan images were imported into Mimics software. The tibia and fibula were extracted with the CT-bone function in Mimics software, and 3D models of the tibia and fibula were obtained. Each model was imported into 3-MATIC and LS-DYNA software to smooth the surface, perform meshing, define material properties, and set the failure parameters, interface properties, vertical loads and boundary conditions. The tibial fractures in both models were calculated to evaluate the biomechanical role of the fibula in the lower leg.

Results

The validity of the model was verified, and the fibular load condition was similar to those previously reported. In the model with fibular support, the fracture load of the tibia was $78.27 \pm 3.36 \mathrm{KN}$, the initial fracture time was $0.69 \pm 0.01 \mathrm{~s}$, and the complete fracture time was $0.75 \pm 0.01 \mathrm{~s}$. The fibula carried approximately $7 \%$ of the load on the lower leg. In the fibular defect model, the fracture load of the tibia was $72.71 \pm 3.25 \mathrm{KN}$, the initial fracture time was $0.54 \pm 0.02 \mathrm{~s}$, and the complete fracture time was $0.62 \pm 0.01 \mathrm{~s}$.

\section{Conclusions}

The fibula play an essential biomechanical role in lower limb load-bearing and therefore is worthy of clinical attention. We believe that LS-DYNA can be used as an effective tool for the study of fracture mechanics.

\section{Background}

With advances in sports medicine, the biomechanical characteristics of the fibula and its role in maintaining the stability of the knee and ankle joints have received increasing attention[1]. However, the fibula was long generally believed to have a negligible role in lower limb load-bearing, as compared with that of the tibia, and was presumed to be a degenerative remnant of evolution[2]. Partial fibular resection is often used as a treatment for chronic osteomyelitis, fibular tumors and other diseases. Fibular grafting is used to treat various bone defects and nonunion because of its high fusion rate. In recent years, with 
the long-term follow-up of lower limb function in patients with fibular defects, morphological changes have been found to occur in the tibia secondarily because of the loss of the fibula[3]. Subsequently, knee weakness, ankle pain and other complications result[4]. These findings suggest that the fibula plays an important biomechanical role in the lower limbs[5].

The mechanical environments of the human fibula and tibia differ greatly[6]. The tibia is designed to translate the compressive load of the whole body weight from two horizontal articular surfaces in the knee to a single horizontal surface in the heel, and to support relatively large additional bending and torsion stresses toward the midshaft [7]. In contrast, the fibula is firmly attached to the proximal tibia by ligaments and is free of weight bearing articular surfaces distally. Some studies have indicated that the fibula contributes a substantial ability to resist axial loading[8-9]. The contribution of the fibula to supporting axial loads is influenced by the complexity of the usual loading patterns and its anatomical relationships with the tibia [3]. Some experts believe that the fibula's contribution to total load may be 11$25 \%$ in humans[9]. All the above studies were concluded through statistical analysis or biomechanical testing of cadaver specimens. To date, studies of the bone and related biomechanics have mainly focused on the static analysis of bone mass and strength, whereas studies combining fracture mechanics,

i.e., bone trabecular microinjury-crack propagation-microfracture-fracture remain scarce. Continuous damage mechanics, which can directly analyze the stress and failure processes of components, is a major topic in the field of engineering mechanics. Through a fracture mechanics simulation of tibial fracture under an axial load, this study compared the differences in dynamic processes in tibial fracture with or without fibular support to explore the biomechanical role of the fibula in lower limbs.

\section{Materials And Methods}

- We retrospectively collected knee joint CT scans from eight adults who had undergone continuous slice CT scanning at the imaging research center of our hospital between June 2020 and December 2020. Patients were excluded if they had a history of lower limb trauma, severe deformities or any underlying diseases. This study was approved by the institutional review board of our hospital after the patients provided informed consent. The mean age of the patients was $45.96 \pm 15.42$ years (range: 30-63 years). DICOM-formatted CT scan images for each patient were imported into Mimics software. The separated 3D reconstruction of the bone structures was acquired with a CT bone segmentation operation. Then the 3D model was generated and smoothed with the 3D calculate function to obtain the initial 3D finite element model (Fig. 1).

The 3D finite element model was imported into 3-matic software for meshing. The tetrahedral meshing method was used, with a mesh size defined as $1.5 \mathrm{~mm}$. We derived the normal model and the fibular defect model with the volume mesh from 3-matic. We then imported the model into LS-DYNA. We set the bone tissue structure and articular cartilage according to previously reported findings [10]. The elastoplastic follow-up strengthening model * MAT_PLASTIC_Kinematic Studio was selected as the 
skeleton material. The main material parameters were as follows: density: 1,900 kg/m3, Poisson's ratio: 0.21, elastic modulus: $2.3 \mathrm{GPa}$, failure strain FS: 0.0204 and solid element Solid164 to simulate the bone.

The data were imported into the large finite element analysis software LS-DYNA to calculate the fracture model of tibial fracture on the basis of the stress-displacement curve relationship. These curves were compared with the fracture lines in the fibular defect model to evaluate fibular support.

The collected data were analyzed in SPSS 25.0 statistical software. The data are represented as mean \pm SD. The t-tests were used to compare the data. The threshold for statistical significance was $P<0.05$.

\section{Results}

The study participants included four men and four women between 30 and 63 years of age, with a mean age of $45.96 \pm 15.42$ years. As shown in Fig. 2, a 3D finite element model of the tibia and fibula was created, which contained 188,011 elements and 272,272 nodes; the fibular defect model contained 165,165 elements and 234,756 nodes. The finite element model of the tibia and fibula was based on the original CT data from patients. During the modeling, operations such as threshold division were automatically completed in Mimics software, with few manual operations, thus avoiding the interference of human factors and better reflecting the fracture situation in patients.

As shown in Table 1, in the normal model, the ultimate tibial load was $78.27 \pm 3.36 \mathrm{KN}$, and in the fibular defect model, the ultimate tibial load was $72.71 \pm 3.25 \mathrm{KN}$. The fibular load accounted for approximately $7 \%$ of the tibia (Fig. 3,4), similar to previously reported findings[11]. In the fibular defect model, the ultimate tibial load was $72.71 \pm 3.25 \mathrm{KN}$, which was significantly lower than the $78.27 \pm 3.36 \mathrm{KN}$ in the normal model $(P<0.05)$. In the fibular defect model, the initiation time of the tibial crack was $0.54 \pm 0.02$ $\mathrm{s}$, and the complete fracture time was $0.62 \pm 0.01 \mathrm{~s}$, whereas in the normal model, the initiation time of the tibial crack was $0.69 \pm 0.01 \mathrm{~s}$, and the complete fracture time was $0.75 \pm 0.01 \mathrm{~s}$ (Fig. 3,4); the difference was significant $(P<0.05)$. The fracture time of the normal model was significantly longer than that of the defect model, thus indicating that the tibia was more prone to fracture in the absence of the fibula, and the complete fracture occurred more quickly. 
Table 1

Comparison of the load, the initiation crack time, and ultimate crack time

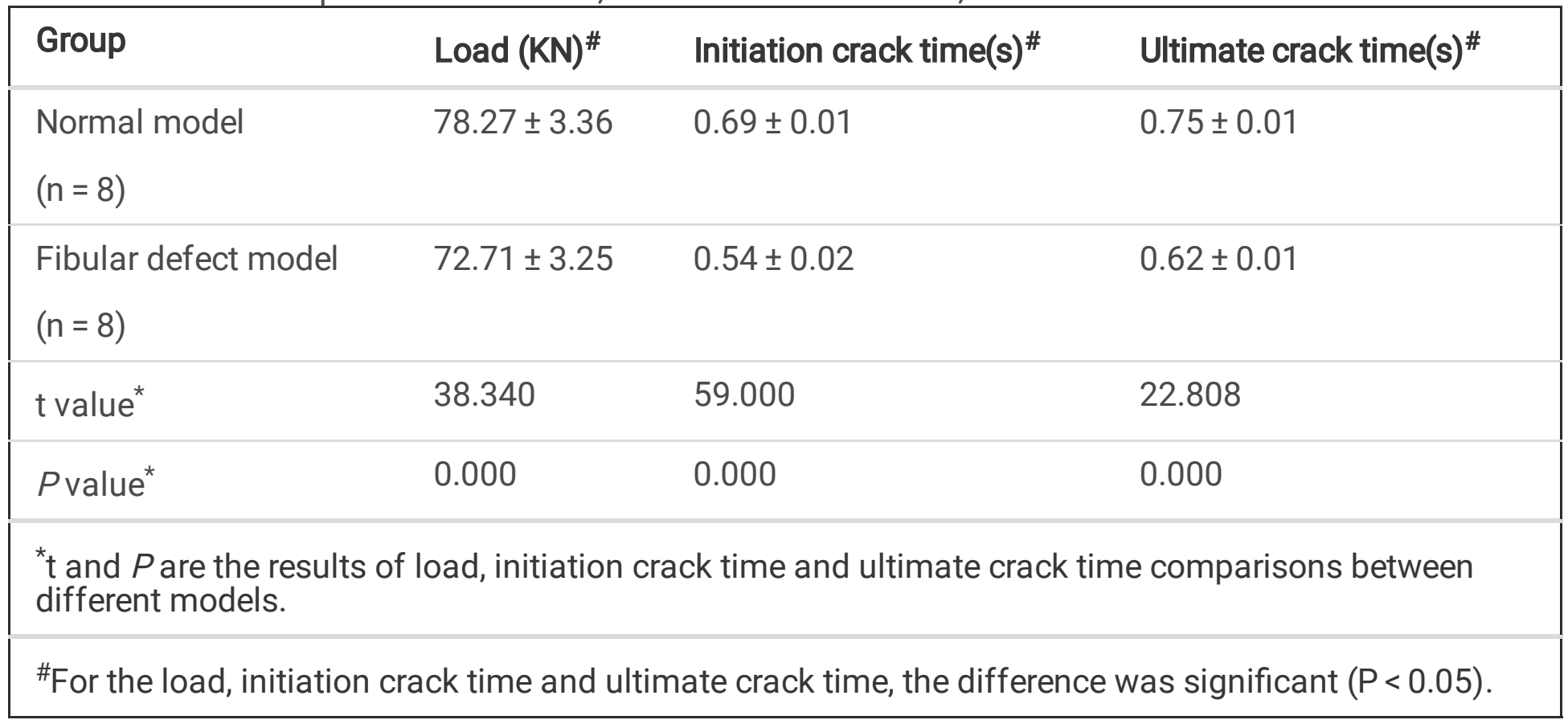

We analyzed the stress nephograms of eight pairs of models and drew the following conclusions. As shown in the following stress nephogram (Fig. 3, 4), in both models, the axial load on the tibia appeared to have a stress concentration in the middle and lower third of the tibia. In the normal model, stress concentration also appeared on the tibiofibular contact surface and the distal end of the fibula. In both models, the crack initiation occurred in the middle and lower third of the tibia, and started from the front of the tibia. The crack propagation then followed the Von Mises equivalent stress distribution to complete fracture in the posterior region of the tibia. The crack propagation path and crack strike formed by the two models were essentially the same. The angle between the crack strike and the horizontal line was approximately $30^{\circ}$, forming an approximately concave curve. These findings indicate that under axial load, the middle and lower third of the tibia have the greatest likelihood of fracture and are the part of the tibia most vulnerable to fracture. The presence of the fibula can delay the occurrence of tibial fracture, but after fractures occurred, the presence of the fibula did not affect the direction and expansion of the bone crack. On the basis of the stress-time curves, we also found that the trend of tibial fracture was essentially linear, and was not associated with the presence or absence of fibular support.

\section{Discussion}

In recent years, with advances in sports medicine, researchers have paid increasing attention to the biomechanical effects of the fibula. Domestic and foreign researchers have conducted relevant studies on the biomechanical effects of the fibula in lower limbs[9, 11-15]. Zahn RK et al. [9] have demonstrated that in patients with severe osteoporosis, the weight bearing of the fibula is critical, owing to the diminished bone quality in older people. Moreover, the authors confirmed the advantage of an internal fixation method that restores the stability of the distal fibula in patients with osteoporosis with distal fibular fractures. Jabara et al.[11] have explained the importance of the stability of the fibular and 
proximal tibiofifibular joint. They have emphasized that neglecting proximal tibiofifibular joint instability may be the reason for the failure of a reconstruction of the posterolateral corner or knee ligament. In studying the proximal tibiofibular joint, Calabro et al.[12-14] have found that the fibula had roles in loadbearing and dispersing the torsion stress on the lower limb. Moreover, patients with proximal tibiofibular joint dislocation have complications such as knee pain and weakness. Alves-da-Silva T et al.[15] have described the kinematics of the proximal tibiofibular joint and its relationship to ankle and knee movements in an exploratory cadaver study.

According to a study by Morin, combined fractures of the distal of tibia and fibula are a common orthopedic injury[16]. Javdan et al.[17] have described that $77.7 \%$ of fibular fractures occur together with tibial fractures. However, the necessity of fibular fixation in fibular and distal tibial fractures remains controversial. Strauss et al.[18] have examined the effects of fibular fixation in distal tibialfibular fractures, particularly distal tibial fractures, in both laboratory and clinical settings; their results have verified that fibular fixation is helpful in maintaining tibial fracture reduction. Previous studies have shown that effective fixation of fibular fractures improves the force line after internal fixation of tibial fractures and decreases tibial reduction failure[19-20]. Elhence et al.[21] have recommended fibular fixation for all distal fractures when two fractures are in the same plane, and the tibial fracture is relatively stable. However, Rouhani et al.[22] have concluded that there was no advantage of fixation of the fibula in the treatment outcomes of tibial diaphysis distal third fractures. However, the literature on the application of fracture mechanics to study the biomechanical effects of the fibula in lower limbs is scarce.

In recent decades, researchers have performed many experiments and studies on fracture problems[2325], thus resulting in the emergence and development of fracture mechanics. At present, finite element analysis is used to study fracture, mainly by considering the mechanism of fracture after external force from falling, and by calculating the Von Mises equivalent stress and combining it with fracture failure criteria. However, the basis for judgment of Von Mises equivalent stress is limited to the starting point of fracture failure, which does not fully reflect the actual fracture situation. LS-DYNA software, the most widely used universal explicit dynamic analysis program worldwide, can simulate various complex problems in the real world and is particularly suitable for solving nonlinear dynamic impact problems of various nonlinear structures. At present, LS-DYNA software is widely used in the field of dynamic analysis, and is even used in the simulation of muscle active response force. Lin et al.[23] have described the influence of regional differences in bone mineral density on hip fracture sites, on the basis of fracture mechanics.

In our research, we found that the fibula carries approximately $7 \%$ of the axial load on the lower leg. A study using a biostatic model has found that the fibula bears one-sixth of the weight on the lower leg [9]. The results of Trainotti et al.[11] have shown that the fibula bore approximately $6.4 \%$ of the body weight. We believe that the differences in the results were due to differences in measurement methods. 
We additionally compared the normal model with the fibular defect model and found that tibias with fibular defects were more prone to fracture, and complete fracture occurred faster. Under axial loading, the fibula can disperse stress and delay the time of tibial fracture, although the presence of the fibula does not affect the location of tibial stress concentration, and essentially does not affect the direction and development of cracks.

In addition, Fan et al.[26] have reported that distal tibial fractures accounted for $37.8 \%$ of all tibial fractures. They believed that fractures of the distal tibia typically occurred because of axial and rotational forces on the lower extremity. In our research, this hypothesis was confirmed by the typical distal fracture of the tibia after axial loading.

There were some limitations to this study. In this fracture analysis, only the load vector was set; the simulation of fractures caused by different external forces would be more helpful to understand the mechanism of tibial fracture. In addition, this study performed a mechanical comparison in only middleaged people with normal bones. Bone mineral density and bone strength can affect the fracture type and stress distribution[27]. Therefore, further study is needed in patients with osteoporosis. More biomechanical studies and related clinical research should be performed.

\section{Conclusion}

Because the fibula plays an essential biomechanical role in the lower limbs, more attention should be paid to the fibula and the complications caused by its absence in clinical settings. We believe that fracture mechanics is indispensable in the study of biomechanical interactions between bones and therefore is worthy of further application.

\section{Abbreviations}

CT: Computed tomography; DICOM: Digital Imaging and Communication in Medicine; 3-D: Threedimensional;

\section{Declarations}

\section{Acknowledgements}

Not applicable.

\section{Authors' contributions}

BS, WDM performed the study, analyzed the data, and drafted the manuscript. DL, JQX and DWW contributed to discussion of data, writing, and editing of the article. TGW and BZ contributed to conception and study design, and editing of the article. All authors read and approved the final manuscript. All authors have read the journal policies and have no issues relating to journal policies. All 
authors have seen the manuscript and approved to submit to your journal. The work described has not been submitted elsewhere for publication, in whole or in part.

\section{Funding}

No funding.

\section{Availability of data and materials}

The datasets generated and analyzed during the current study are available from the corresponding author on reasonable request.

\section{Ethics approval and consent to participate}

This study has obtained ethics approval and consent of the ethics committee in our hospital.

\section{Consent for publication}

Not applicable.

\section{Competing interests}

The authors declare that they have no conflict of interest.

\section{References}

1. Cheng JW, Li ZJ, Wei QJ. Study on the effect of fibula on the stability of ankle and knee joint[J]. Journal of Guangxi Medical University, 2020, 37(5): 956-961.

2. Lambert KL. The weight-bearing function of the fibula. A strain gauge study[J]. J Bone Joint Surg Am. 1971;53(3):507-513.

3. Taddei F, Balestri $M$, Rimondi $E$, et al. Tibia adaptation after fibula harvesting: an in vivo quantitative study[J]. Clin Orthop Relat Res. 2009;467(8):2149-2158.

4. Bodde EW, De VE, Duysens JE, et al. Donor-site morbidity after free vascularized autogenous fibular transfer: subjective and quantitative analyses[J]. Plast Reconstr Surg. 2003; 111(7): 2237-2242.

5. Anavian J, Marchetti DC, Moatshe G, et al. The forgotten joint: quantifying the anatomy of the proximal tibiofibular joint[J]. Knee Surg Sports Traumatol Arthrosc. 2018;26(4):1096-1103.

6. Cointry GR, Nocciolino L, Ireland A, et al. Structural differences in cortical shell properties between upper and lower human fibula as described by PQCT serial scans. A biomechanical interpretation[J]. Bone. 2016;90:185-194.

7. Rantalainen T, Nikander R, Heinonen A, et al. Direction-specific diaphyseal geometry and mineral mass distribution of tibia and fibula: a pQCT study of female athletes representing different exercise loading types[J]. Calcif Tissue Int. 2010;86(6):447-454. 
8. Susanne T, Stefan R, Marco K, et al. Locking versus nonlocking plates in mandibular reconstruction with fibular graft-a biomechanical ex vivo study[J]. Clinical Oral Investigations, 2014,18(4).

9. Zahn RK, Frey S, Jakubietz RG, et al. A contoured locking plate for distal fibular fractures in osteoporotic bone: a biomechanical cadaver study[J]. Injury. 2012;43(6):718-725.

10. Wu Q, Ma L, Liu Q, et al. Impact response and energy absorption of human skull cellular bones[J]. J Mech Behav Biomed Mater. 2018;81:106-119.

11. Jabara M, Bradley J, Merrick M. Is stability of the proximal tibiofibular joint important in the multiligament-injured knee?[J]. Clin Orthop Relat Res. 2014;472(9):2691-2697.

12. Calabró $T$, Cevolani $L$, Chehrassan $M$, et al. A new technique of reduction for isolated proximal tibiofibular joint dislocation: a case report[J]. Eur Rev Med Pharmacol Sci. 2014;18(1 Suppl):93-95.

13. Van AF, Musters L, Sonnega RJ, et al. Dislocation of the proximal tibiofibular joint, do not miss it[J]. BMJ Case Rep. 2015; 2015: bcr2014207875.

14. Haupt S, Frima $H$, Sommer C. Proximal tibiofibular joint dislocation associated with tibial shaft fractures - 7 Cases[J]. Injury. 2016;47(4):950-953.

15. Teresa A, Francisco GP, Ricardo M, et al. Kinematics of the proximal tibiofibular joint is influenced by ligament integrity, knee and ankle mobility: an exploratory cadaver study[J]. Knee Surgery Sports Traumatology Arthroscopy, 2018, 27.

16. Morin PM, Reindl R, Harvey EJ, et al. Fibular fixation as an adjuvant to tibial intramedullary nailing in the treatment of combined distal third tibia and fibula fractures: a biomechanical investigation[J]. Can J Surg. 2008;51(1):45-50.

17. Javdan M, Tahririan MA, Nouri M. The Role of Fibular Fixation in the Treatment of Combined Distal Tibia and Fibula Fracture: A Randomized, Control Trial[J]. Adv Biomed Res. 2017;6:48. Published 2017 Apr 25.

18. Strauss EJ, Alfonso D, Kummer FJ, et al. The effect of concurrent fibular fracture on the fixation of distal tibia fractures: a laboratory comparison of intramedullary nails with locked plates[J]. J Orthop Trauma. 2007;21(3):172-177.

19. Egol KA, Weisz R, Hiebert $R$, et al. Does fibular plating improve alignment after intramedullary nailing of distal metaphyseal tibia fractures?[J]. J Orthop Trauma. 2006;20(2):94-103.

20. Kumar A, Charlebois SJ, Cain EL, et al. Effect of fibular plate fixation on rotational stability of simulated distal tibial fractures treated with intramedullary nailing[J]. J Bone Joint Surg Am. 2003;85(4):604-608.

21. Elhence A, Jalan D, Talreja $\mathrm{H}$. To fix or not to fix? The role of fibular fixation in distal shaft fractures of the leg[J]. Injury. 2014;45(11):1802-1803.

22. Rouhani A, Elmi A, Aghdam HA, et al. The role of fibular fixation in the treatment of tibia diaphysis distal third fractures[J]. Orthopaedics \& Traumatology: Surgery \& Research, 2012, 98(8):868-872.

23. Lin ZL, Li PF, Pang ZH, et al. Influence of Regional Difference in Bone Mineral Density on Hip Fracture Site in Elderly Females by Finite Element Analysis[J]. Cell Biochem Biophys. 2015;73(2):405-412. 
24. Hambli R, Allaoui S. A robust 3D finite element simulation of human proximal femur progressive fracture under stance load with experimental validation[J]. Ann Biomed Eng. 2013;41(12):25152527.

25. Seref-Ferlengez Z, Kennedy OD, Schaffler MB. Bone microdamage, remodeling and bone fragility: how much damage is too much damage?[J]. Bonekey Rep. 2015;4:644.

26. Fan $\mathrm{CY}$, Chiang CC, Chuang TY, et al. Interlocking nails for displaced metaphyseal fractures of the distal tibia[J]. Injury. 2005;36(5):669-674.

27. Koester KJ, lii J, Ritchie RO. The true toughness of human cortical bone measured with realistically short cracks[J]. Nat Mater. 2008;7(8):672-677.

\section{Figures}
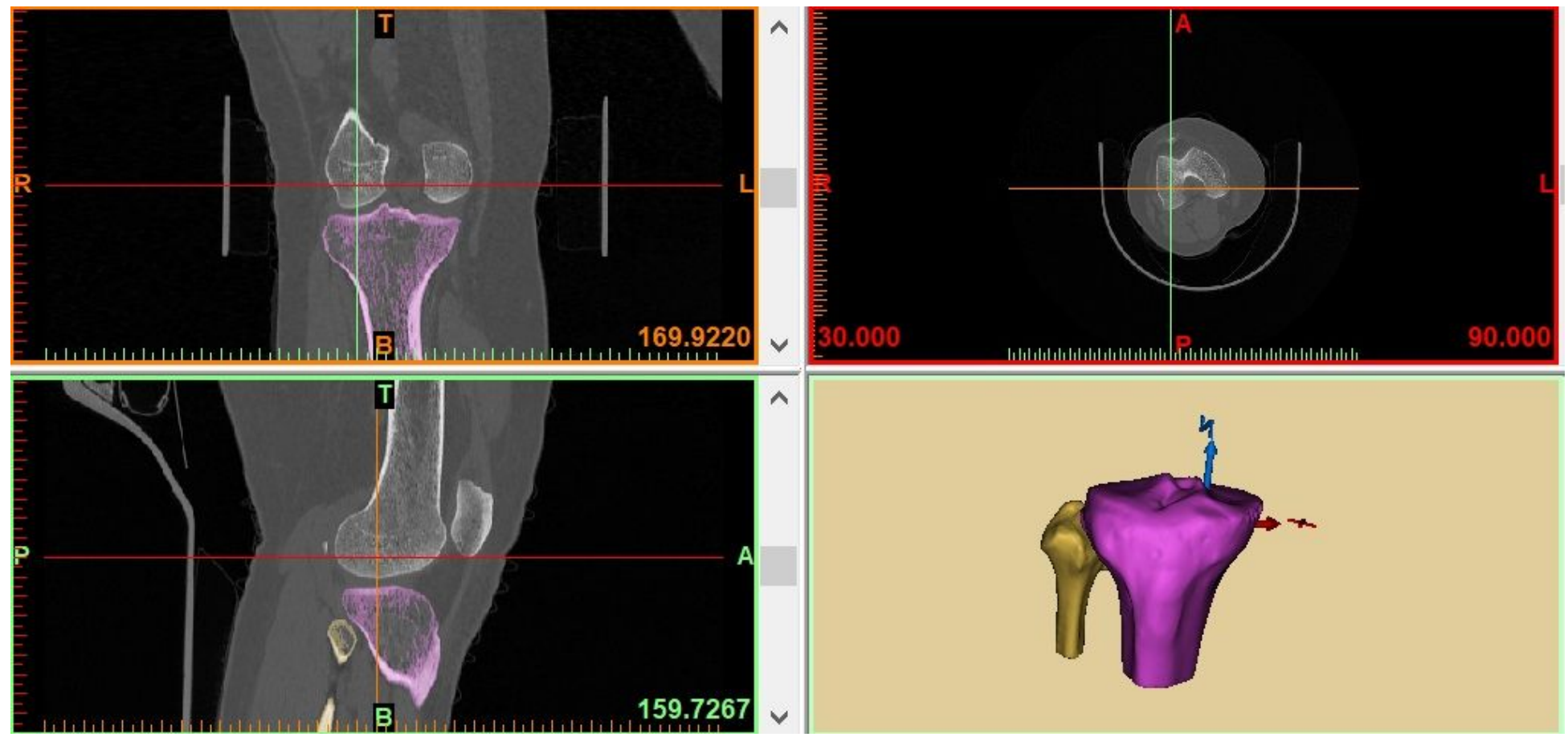

\section{Figure 1}

DICOM-formatted CT scan images were imported into Mimics software. The 3D model was created with image CT-bone and calculate 3D. 
A

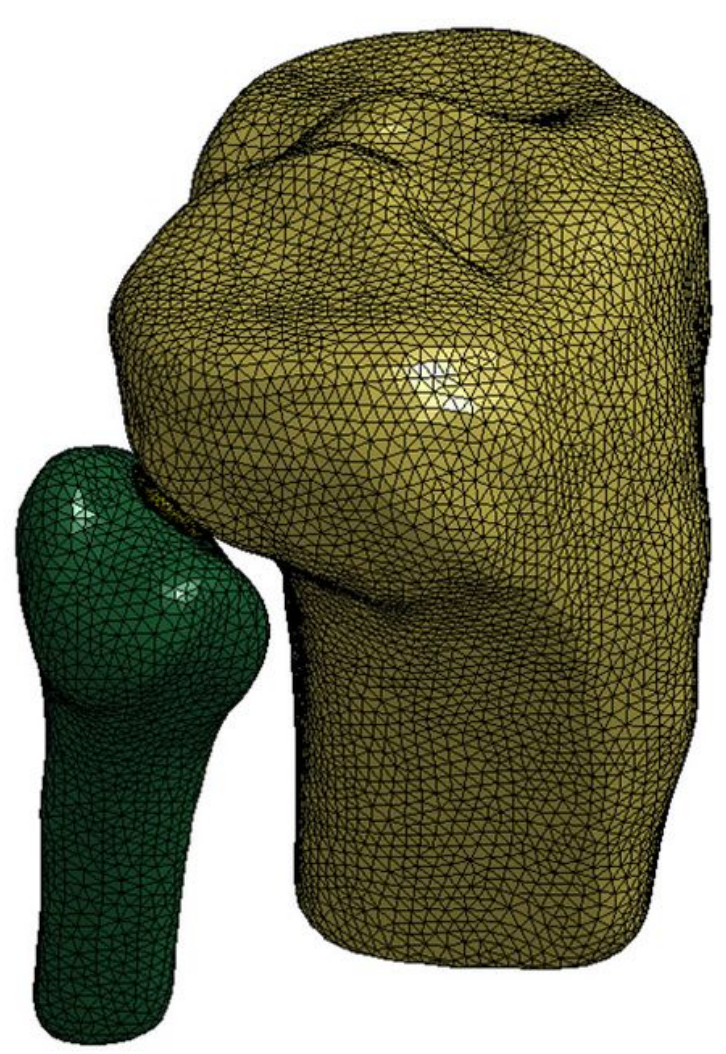

B

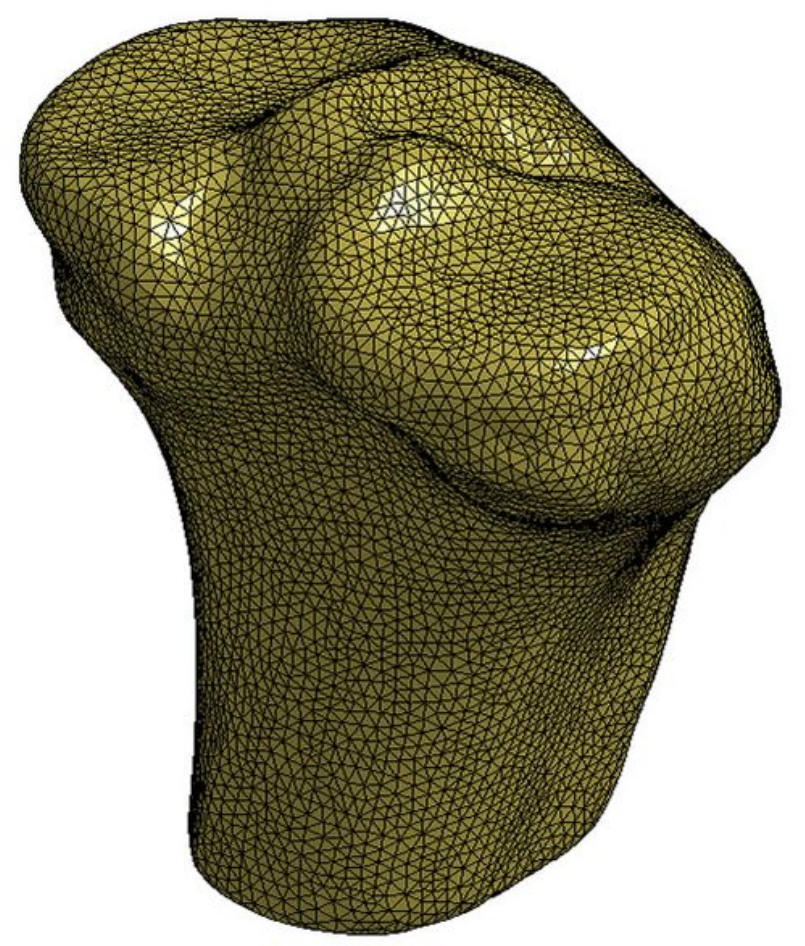

Figure 2

The models with volume mesh. A Normal model. B Fibular defect model. 

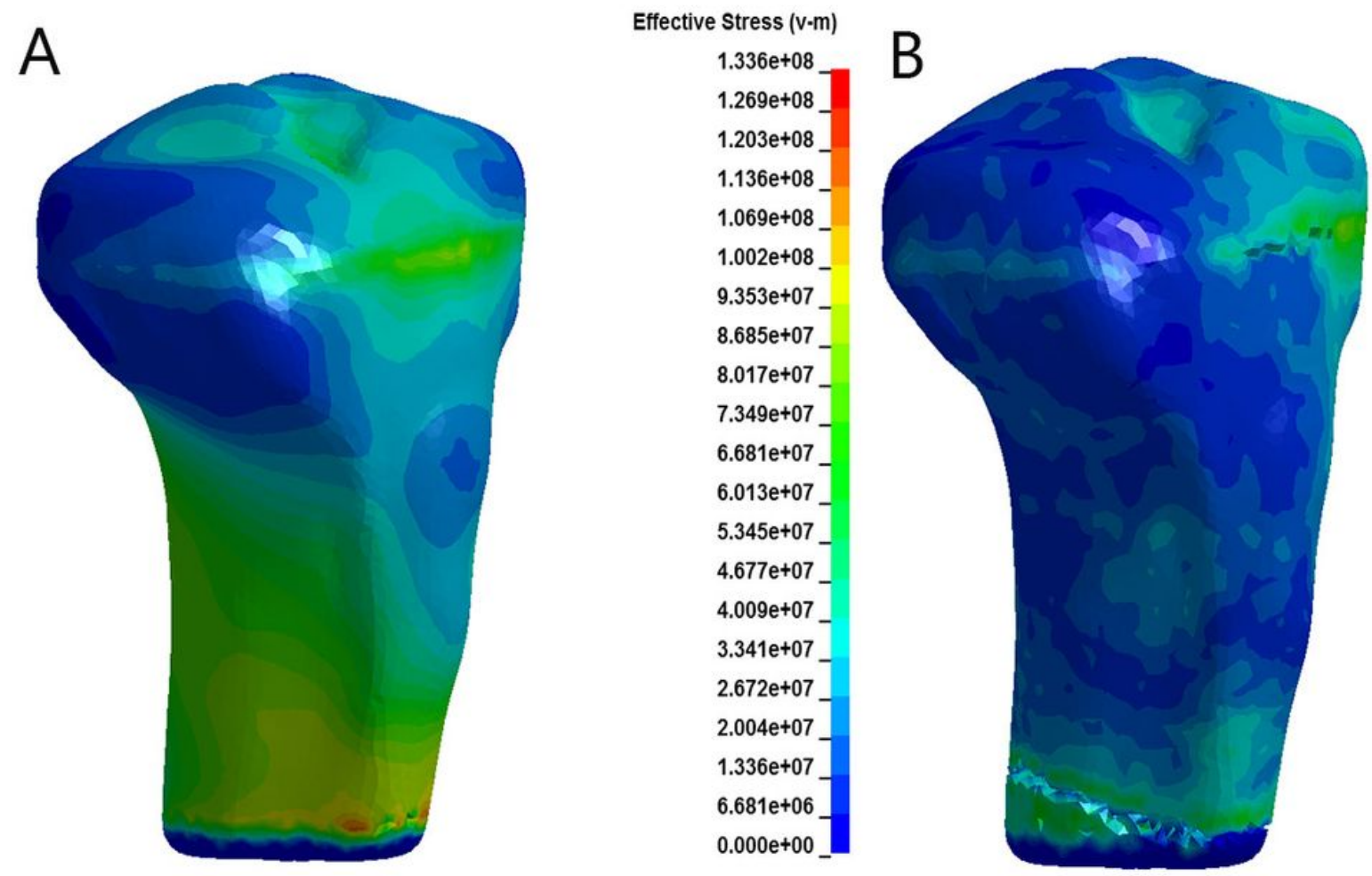

Effective Stress (v-m)

$7.825 \mathrm{e}+07$
$7.434 \mathrm{e}+07$
$7.042 \mathrm{e}+07$
$6.651 \mathrm{e}+07$
$6.260 \mathrm{e}+07$
$5.869 \mathrm{e}+07$
$5.477 \mathrm{e}+07$
$5.086 \mathrm{e}+07$
$4.695 \mathrm{e}+07$
$4.304 \mathrm{e}+07$
$3.912 \mathrm{e}+07$
$3.521 \mathrm{e}+07$
$3.130 \mathrm{e}+07$
$2.739 \mathrm{e}+07$
$2.347 \mathrm{e}+07$
$1.956 \mathrm{e}+07$
$1.565 \mathrm{e}+07$
$1.174 \mathrm{e}+07$
$7.825 \mathrm{e}+06$
$3.912 \mathrm{e}+06$
$0.000 \mathrm{e}+00$

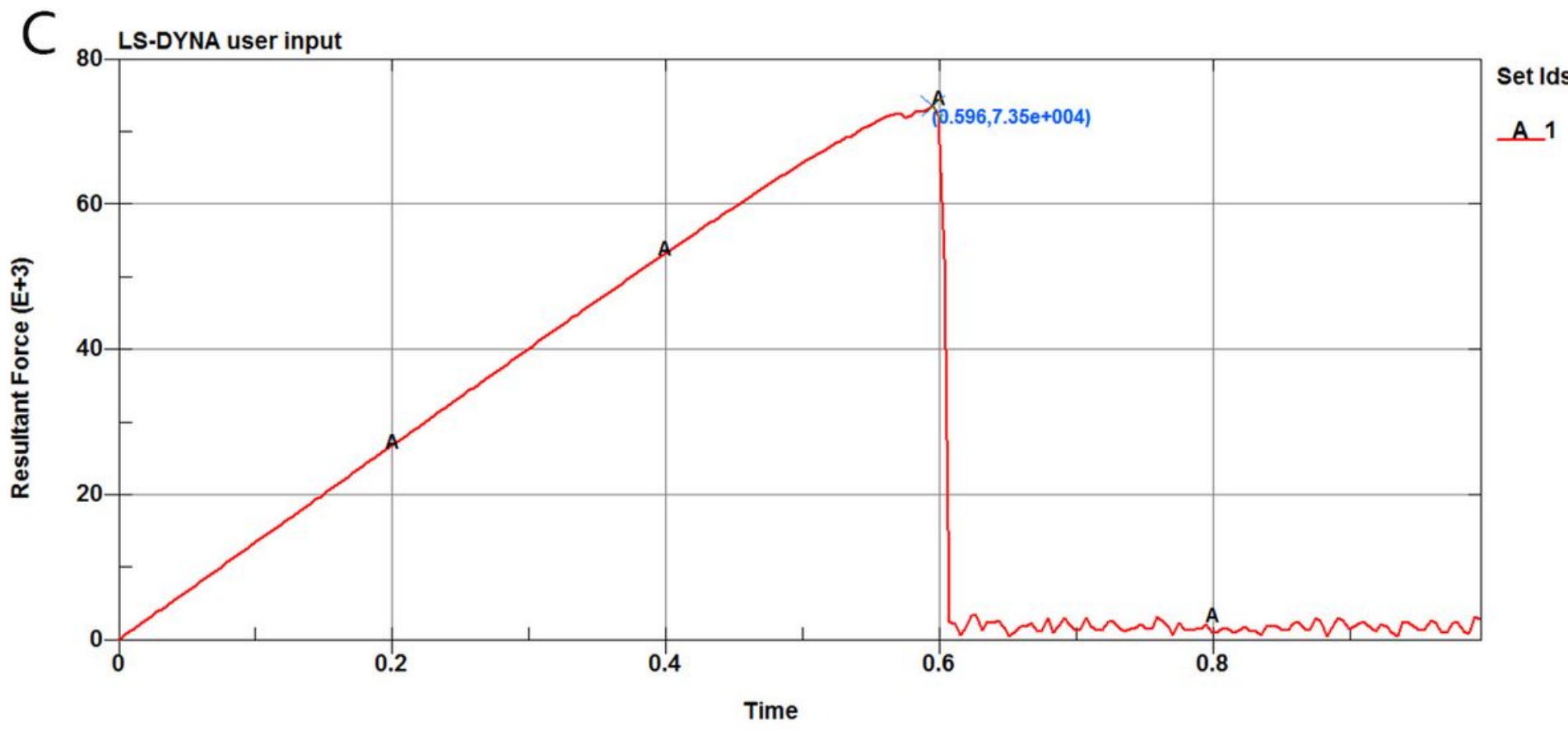

\section{Figure 3}

Fibular defect model. A. Stress nephogram of the tibia at the beginning of fracture. B. Stress nephogram of the tibia at complete fracture. C. The fracture load of the tibia was $72.71 \pm 3.25 \mathrm{KN}$, the time of tibial crack initiation was $0.54 \pm 0.02 \mathrm{~s}$, and the time of complete fracture of the tibia was $0.62 \pm 0.01 \mathrm{~s}$. 
A

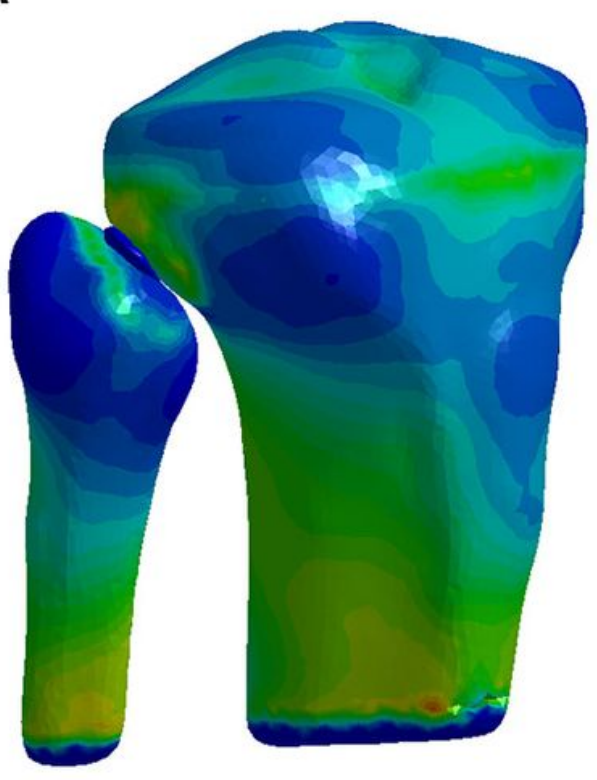

Effective Stress (v-m)

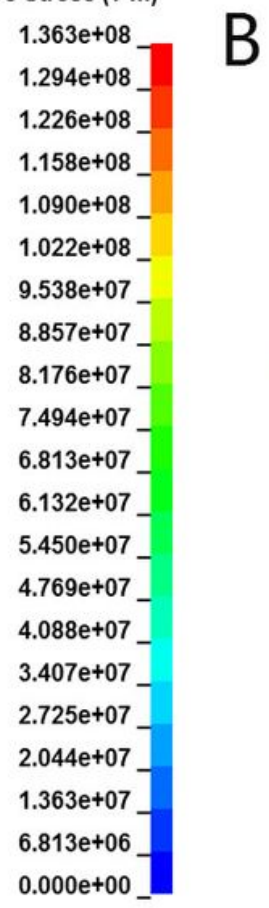

Effective Stress (v-m)

$1.398 \mathrm{e}+08$

$1.328 \mathrm{e}+08$

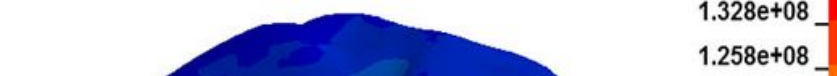

$1.188 \mathrm{e}+08$

$1.119 \mathrm{e}+08$

$1.049 \mathrm{e}+08$

$9.787 \mathrm{e}+07$

$9.088 \mathrm{e}+07$

$8.389 \mathrm{e}+07$

$7.690 \mathrm{e}+07$

$6.991 \mathrm{e}+07$

$6.292 \mathrm{e}+07$

$5.593 \mathrm{e}+07$

$4.894 \mathrm{e}+07$

$4.194 \mathrm{e}+07$

$3.495 \mathrm{e}+07$

$2.796 \mathrm{e}+07$

$2.097 \mathrm{e}+07$

$1.398 \mathrm{e}+07$

$6.991 \mathrm{e}+06$

$0.000 \mathrm{e}+00$

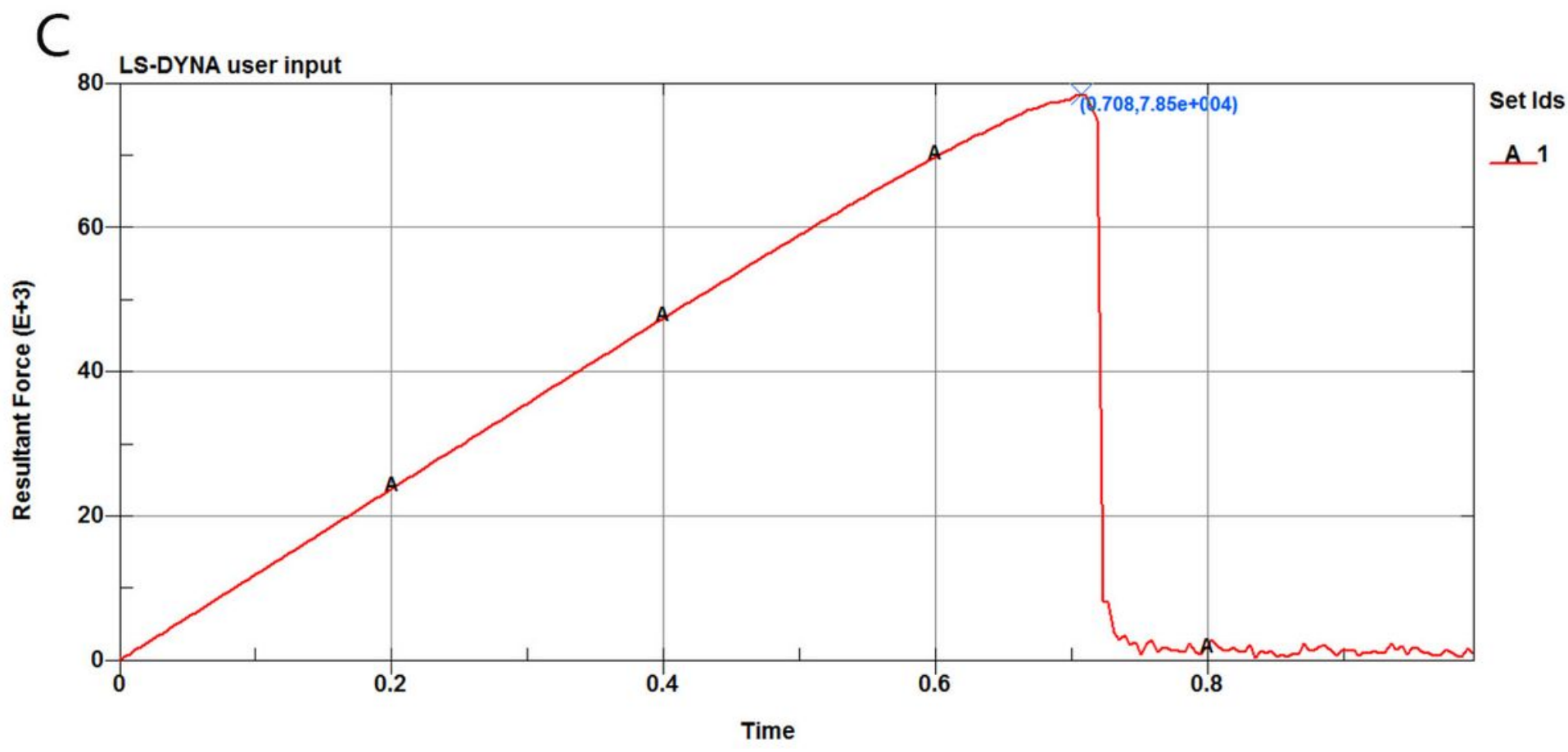

\section{Figure 4}

The normal model. A. Stress nephogram of the tibia at the beginning of fracture. B. Stress nephogram of the tibia at complete fracture. C. The fracture load of the tibia was $78.27 \pm 3.36 \mathrm{KN}$, the time of tibial crack initiation was $0.69 \pm 0.01 \mathrm{~s}$, and the time of complete fracture of the tibia was $0.75 \pm 0.01 \mathrm{~s}$. 